\title{
CAPM and APT-like models with risk measures
}

\author{
Alejandro Balbás ${ }^{\mathrm{a}, *}$, Beatriz Balbás ${ }^{\mathrm{b}}$, Raquel Balbás ${ }^{\mathrm{c}}$ \\ a University Carlos III of Madrid, C/ Madrid, 12628903 Getafe, Madrid, Spain \\ ${ }^{b}$ University Rey Juan Carlos, Paseo de Artilleros s/n, 28032 Madrid, Spain \\ ${ }^{c}$ University Complutense of Madrid, Department of Actuarial and Financial Economics, Somosaguas Campus, 28223 Pozuelo, Madrid, Spain
}

\section{A R T I C L E I N F O}

\section{Article history:}

Received 11 June 2009

Accepted 11 November 2009

Available online 16 November 2009

\section{JEL classification:}

G11

G13

Keywords:

Risk measure

Compatibility between prices and risks

Efficient portfolio

APT and CAPM-like models

\begin{abstract}
A B S T R A C T
The paper deals with optimal portfolio choice problems when risk levels are given by coherent risk mea sures, expectation bounded risk measures or general deviations. Both static and dynamic pricing models may be involved. Unbounded problems are characterized by new notions such as (strong) compatibility between prices and risks. Surprisingly, the lack of bounded optimal risk and/or return levels arises for important pricing models (Black and Scholes) and risk measures (VaR, CVaR, absolute deviation, etc.) Bounded problems present a Market Price of Risk and generate a pair of benchmarks. From these bench marks we introduce APT and CAPM like analyses, in the sense that the level of correlation between every available security and some economic factors explains the security expected return. The risk level non correlated with these factors has no influence on any return, despite the fact that we are dealing with risk functions beyond the standard deviation.
\end{abstract}

\section{Introduction}

General risk functions are becoming very important in finance and insurance. Since Artzner et al. (1999) introduced the axioms and properties of the "Coherent Measures of Risk" many authors have extended the discussion. The recent development of new markets (insurance or weather linked derivatives, commodity derivatives, energy/electricity markets, etc.) and products (infla tion linked bonds, equity indexes annuities or unit links, hedge funds, etc.), the necessity of managing new types of risk (credit risk, operational risk, etc.) and the (often legal) obligation of pro viding initial capital requirements have made it necessary to over come the variance as the most used risk measure and to introduce more general risk functions. It has been proved that the variance is not compatible with the Second Order Stochastic Dominance if asymmetries and heavy tails are involved (Ogryczak and Ruszczyn ski, 1999).

Hence, it is not surprising that the recent literature presents many interesting contributions focusing on new methods for mea suring risk levels. Among others, Föllmer and Schied (2002) have defined the Convex Risk Measures, Goovaerts et al. (2004) have introduced the Consistent Risk Measures, Rockafellar et al.

\footnotetext{
* Corresponding author. Tel.: +34 916249636; fax: +34 916249606.

E-mail addresses: alejandro.balbas@uc3m.es (A. Balbás), beatriz.balbas@urjc.es (B. Balbás), raquel.balbas@ccee.ucm.es (R. Balbás).
}

(2006a) have defined the General Deviations and the Expectation Bounded Risk Measures, and Brown and Sim (2009) have intro duced the Satisfying Measures.

Many classic actuarial and financial problems have been revis ited using new risk functions. For instance, pricing and hedging is sues in incomplete markets (Föllmer and Schied, 2002; Nakano, 2004; Staum, 2004; Balbás et al., 2010, etc.), as well as equity linked annuities hedging issues (Barbarin and Devolder, 2005), optimal reinsurance problems (Balbás et al., 2009), portfolio insur ance linked problems (Annaert et al., 2009) and other practical topics.

With regard to portfolio choice and asset allocation problems, among others, Alexander et al. (2006) compare the minimization of the Value at Risk ( VaR) and the Conditional Value at Risk $(\mathrm{CVaR})$ for a portfolio of derivatives (such a portfolio is obviously com posed of asymmetric securities and, therefore, the standard devia tion is not appropriate), Calafiore (2007) studies "robust" efficient portfolios in discrete probability spaces if the risk measure is the absolute deviation, Schied (2007) focuses on optimal investment with convex risk measures, Quaranta and Zaffaroni (2008) studies "robust" optimization of the VaR, Zhiping and Wang (2008) deals with "two sided" coherent risk measures and optimal portfolios, and Miller and Ruszczynski (2008) analyze efficient portfolios with coherent risk measures. Other authors have also dealt with gener alizations of the Sharpe ratio, the introduction of benchmarks along the lines of the Market Portfolio of the classic Capital Asset 
Pricing Model (CAPM), and the extension of formulas relating ex pected returns to some kind of generalized betas, also along the lines of the CAPM. For instance, Stoyanov et al. (2007) have intro duced new ratios related to many risk measures such as CVaR, and leading to various benchmark portfolios (see also further extensions of Zakamouline and Koekebbaker, 2009), and Johnston (2009) has combined the classical CAPM with the Asymptotic sin gle Risk Factor approach so as to compute capital requirements for credit and equity exposures. Similarly, Rockafellar et al. $(2006 \mathrm{~b}, \mathrm{c}))$ have analyzed portfolio choice problems when risk lev els are given by deviation measures, have introduced benchmarks, and have defined new betas related to the deviation they are using that preserves the usual relationship between beta and expected return. Rockafellar et al. (2007) have also shown the possible exis tence of equilibrium if agents deal with general deviations.

The present paper considers a general measure of risk $\rho$. Both expectation bounded risk measures and deviations are included in the analysis, as well as coherent risk measures. Then, we present a classic risk/return mathematical programming problem whose solutions are the efficient portfolios. An important novelty is that this portfolio choice problem involves both $\rho$ and the market pric ing rule denoted by $\Pi$. From a theoretical point of view, consider ing $\Pi$ seems to present some advantages with respect to the usual analysis focusing on the distributions of the available assets' re turns. Indeed, $\Pi$ will be characterized by the Stochastic Discount Factor $(S D F) z_{\pi}$ of the economy (Chamberlain and Rothschild, 1983 , or Duffie, 1988) which will permit us to study many proper ties by connecting the SDF $z_{\pi}$ of $\Pi$ and the sub gradient $\Delta_{\rho}$ of $\rho$.

The paper's outline is as follows. Section 2 presents the nota tions and the general framework we are going to deal with. Section 3 will be devoted to studying the properties of the efficient portfo lios. The section is divided into three subsections. In the first one the general portfolio choice problem is discussed, and necessary and sufficient optimality conditions are provided (Theorem 3 ). This seems to be one of the first times that those kinds of conditions are given for maybe infinite dimensional portfolio choice problems. As said above, we use pricing rules rather than return distributions, which allows us to consider dynamic pricing models (the Black and Scholes model, for instance) leading to infinite dimensional optimization problems.

We use Theorem 3 in the second subsection of Section 3 so as to present many cases leading to meaningless economic properties. So, although the notion of compatibility between pricing rules and risk measures has been defined in Balbás and Balbás (2009), this paper deals with its implications in portfolio choice. Theorem 4 shows that risk levels may tend to $\infty$ while expected returns simultaneously tend to $\infty$ if the lack of compatibility applies. We also point out that many important risk measures (VaR, CVaR, weighted CVaR or WCVaR, Dual Power Transform or DPT, etc.) are not compatible with very important pricing models (Black and Scholes, Heston, etc.). All of these cases lead to unbounded risk and returns.

We also introduce the new notion of strong compatibility be tween a pricing rule and a risk measure (Definition 2). Once again, the lack of strong compatibility makes the expected return be un bounded, although the risk level remains bounded in this case. This pathological situation applies for very important compatible risk measures and deviations (the measure of Wang, the Compatible Conditional Value at Risk or CCVaR, the absolute deviation, the absolute down side semi deviation, etc.) along with important pricing models (Black and Scholes, Heston, etc.). Theorem 6 and its remarks clarify this finding, which may be of great interest to managers and traders. Indeed, many risk measures are used in practice so as to compute capital requirements, so an unbounded optimal risk/return problem may provide practitioners with prac tical tools to obtain significant decreases in risk levels and capital requirements, which are sometimes also understood as opportu nity costs. Finally, there are two additional remarkable findings in this subsection. Firstly, we define the new deviation measure $\widetilde{N}$ so as to overcome the incompatibility of the CVaR and the WCVaR with respect to the Black and Scholes model. Secondly, we show that the standard deviation is strongly compatible with every pricing rule.

The third subsection of Section 3 is devoted to those situations presenting strong compatibility. In such a case we will introduce the benchmark and the Capital Market Line $(C M L)$ for a general couple $(\Pi, \rho)$, as well as the Market Price of Risk.

The fourth section of the paper deals with extensions of the classic Arbitrage Pricing Theory (APT) and CAPM. With respect to the important contributions of Rockafellar et al. (2006b) our ap proach seems to present four major novelties. First, it also applies for expectation bounded or coherent risk measures. Second, it also deals with the APT model. Third, it clarifies that this type of analy sis only makes sense in the presence of strong compatibility. And fourth, our betas are essentially different from those of Rockafellar et al. (2006b), and they are similar to those of the classic CAPM and $A P T$ (which uses the standard deviation). We do not use the risk/ deviation measure $\rho$ so as to define the betas. On the contrary, they are given by the covariance between the returns of the analyzed security and the factors explaining the market $(A P T)$ or the bench mark generating the $C M L(C A P M)$. This fact seems to reflect an advantage since one can clearly see that the betas and the system atic risk indicate correlation with the factors/market, while the specific risk and the specific noise have null correlation with the factors/market, and, therefore, cannot be explained by them. In this sense, the betas are reflecting the information about the analyzed security that is given by the factors/market, and the approach be comes quite parallel to that of the classical APT or CAMP. This could be another advantage provided by the use of pricing rules and the $S D F$, a major concept in Financial Economics.

Summarizing, there seems to be several contributions in this paper. First, we provide general optimality conditions in a portfolio choice problem that may involve static and dynamic pricing mod els. Second, we introduce the new notion of strong compatibility between prices and risks and study the effect of both the lack of compatibility and the lack of strong compatibility. We point out that the lack of (strong) compatibility applies in very important Financial Economics models. Third, models with a market price of risk are also characterized and analyzed, and they also may in volve dynamic pricing models. Finally, we present APT and $C A P M$ like developments are for general risk measures, and do not modify the classic definitions of the betas. On the contrary, sys tematic risks depend on the correlations with the factors/market, whereas idiosyncratic risks and noises will be non correlated with them.

Section 5 summarizes the most important conclusions of the paper.

\section{Preliminaries and notations}

Consider the probability space $(\Omega, \mathcal{F}, \mu)$ composed of the set of "states of the world" $\Omega$, the $\sigma$ algebra $\mathcal{F}$ and the probability mea sure $\mu$. If $p \in[1, \infty), L^{p}$ will denote the space of $\mathbb{R}$ valued random variables $y$ on $\Omega$ such that $\mathbb{E}\left(|y|^{p}\right)<\infty$, $\mathbb{E}()$ representing the mathe matical expectation. If $q \in(1, \infty]$ is its conjugate value (i.e., $1 / p+1 / q \quad 1)$, then the Riesz Representation Theorem (Horvàth, 1966) guarantees that $L^{q}$ is the dual space of $L^{p}$, where $L^{\infty}$ is com posed of the essentially bounded random variables. A special important case arises for $p \quad q \quad 2$.

Consider a time interval $[0, T]$, a subset $\mathcal{T} \subset[0, T]$ of trading dates containing 0 and $T$, and a filtration $\left(\mathcal{F}_{t}\right)_{t \in \mathcal{T}}$ providing the 
arrival of information and such that $\mathcal{F}_{0} \quad\{\emptyset, \Omega\}$ and $\mathcal{F}_{T} \quad \mathcal{F}$. We will denote by $Y \subset L^{2}$ a closed subspace composed of reachable pay offs, i.e., if $y \in Y$ there exists an adapted to the filtration $\left(\mathcal{F}_{t}\right)_{t \in \mathcal{T}}$ price process of a self financing portfolio $\left(S_{t}\right)_{t \in \mathcal{T}}$, such that $S_{T} \quad y$, a.s. Then, if $\Pi(y) \quad S_{0}$, following usual conventions we will suppose that the pricing rule

$\Pi: Y \rightarrow \mathbb{R}$

providing us with the price $\Pi(y)$ of every $y \in Y$ is linear and contin uous. ${ }^{1}$ As usual, the market will be said to be complete if $Y L^{2}$.

Assume the existence of a riskless asset. Denote by $r_{f} \geqslant 0$ the risk free rate. The equality

$\Pi(k) \quad k e^{r_{f} T}$

must hold for every $k \in \mathbb{R}$.

Being $Y$ a Hilbert space the Riesz Representation Theorem im plies the existence of a unique $z_{\pi} \in Y$ such that

$\Pi(y) \quad e^{r_{f} T} \mathbb{E}\left(y z_{\pi}\right)$

for every $y \in Y . z_{\pi}$ is usually called "Stochastic Discount Factor" $(S D F)$, and is closely related to the Market Portfolio of the CAPM (Duffie, 1988).

Expression (1) implies that

$k e^{r_{f} T} \Pi(k) \quad e^{r_{f} T} k \mathbb{E}\left(z_{\pi}\right)$,

which leads to

$\mathbb{E}\left(z_{\pi}\right) \quad 1$.

Let $p \in[1,2]$ and

$\rho: L^{p} \rightarrow \mathbb{R}$

be the general risk function that a trader uses in order to control the risk level of his final wealth at $T$. Denote by

$\Delta_{\rho} \quad\left\{z \in L^{q} ; \quad \mathbb{E}(y z) \leqslant \rho(y), \forall y \in L^{p}\right\}^{2}$

The set $\Delta_{\rho}$ is obviously convex. We will assume that $\Delta_{\rho}$ is also $\sigma\left(L^{q}, L^{p}\right)$ compact, ${ }^{3}$ and

$\rho(y) \quad \operatorname{Max}\left\{\mathbb{E}(y z): z \in \Delta_{\rho}\right\}$

holds for every $y \in L^{p}$. Furthermore, we will also impose the exis tence of $\widetilde{E} \in \mathbb{R}, \widetilde{E} \geqslant 0$, such that

$\Delta_{\rho} \subset\left\{z \in L^{q} ; \mathbb{E}(z) \quad \widetilde{E}\right\}$.

Summarizing, we have:

Assumption 1. The set $\Delta_{\rho}$ given by (4) is convex and $\sigma\left(L^{q}, L^{p}\right)$ compact, (5) holds for every $y \in L^{p}, z \quad \widetilde{E}$ a.s. is in $\Delta_{\rho}$ and (6) holds.

The assumption above is closely related to the Representation Theorem of Risk Measures stated in Rockafellar et al. (2006a). Fol lowing their ideas, it is easy to prove that the fulfillment of Assumption 1 holds if and only if $\rho$ is continuous and satisfies:

(a) Translation invariance

$\rho(y+k) \quad \rho(y) \quad \widetilde{E} k$

for every $y \in L^{p}$ and $k \in \mathbb{R}$.

(b) Homogeneity

$\rho(\alpha y) \quad \alpha \rho(y)$

for every $y \in L^{p}$ and $\alpha>0$.

\footnotetext{
1 The absence of arbitrage implies that $S_{0}$ must remain the same if there is more than one self-financing portfolio whose final value equals $y \in Y$.

2 Notice that $\mathrm{q} \in[2, \infty]$.

${ }^{3}$ See Horvàth (1966) for further details about $\sigma\left(L^{q}, L^{p}\right)-$ compact sets.
}

(c) Sub additivity

$\rho\left(y_{1}+y_{2}\right) \leqslant \rho\left(y_{1}\right)+\rho\left(y_{2}\right)$

for every $y_{1}, y_{2} \in L^{p}$.

(d) Mean dominating

$\rho(y) \geqslant \widetilde{E} \mathbb{E}(y)$

for every $y \in L^{p}$. $^{4}$

It is easy to see that if $\rho$ is continuous and satisfies Properties $a$ ), $b), c$ ) and $d$ ) above with $\widetilde{E} \quad 1$ then it is also coherent in the sense of Artzner et al. (1999) if and only if

$\Delta_{\rho} \subset L_{+}^{q} \quad\left\{z \in L^{q} ; \mu(z \geqslant 0) \quad 1\right\}$.

Particularly, interesting examples with $\widetilde{E} \quad 1$ are the Expectation Bounded Risk Measures of Rockafellar et al. (2006a). For instance, the Conditional Value at Risk $(\mathrm{CVaR})$ and the Weighted Conditional Value at Risk (WCVaR) (Rockafellar et al., 2006a), the Compatible Conditional Value at Risk (CCVaR) of Balbás and Balbás (2009), the Dual Power Transform (DPT) of Wang (2000) and the Wang Mea sure (Wang, 2000), among many others. ${ }^{5}$ Particular interesting examples with $\widetilde{E} \quad 0$ are the deviation measures of Rockafellar et al. (2006a). Among others, the classical $p$ deviation given by

$\sigma_{p}(y) \quad\left[\mathbb{E}\left(|\mathbb{E}(y) \quad y|^{p}\right)\right]^{1 / p}$

or the downside $p$ semi deviation given by

$\sigma_{p}(y) \quad\left[\mathbb{E}\left(|\operatorname{Max}\{\mathbb{E}(y) \quad y, 0\}|^{p}\right)\right]^{1 / p}$

which are also particular cases of Orlicz deviation measures (Cheri dito and Tianhui, 2009).

Finally, let us remark that with $L^{2}$ being a Hilbert space there are orthogonal projections on every closed subspace. In particular, we will focus on $\varphi_{Y}$ and $\varphi_{\pi}$, the orthogonal projections on $Y$ and the linear manifold $\mathcal{L}\left(1, z_{\pi}\right) \subset Y$, respectively (see Maurin, 1967, for further details about the orthogonal projection in Hilbert spaces).

\section{Portfolio choice}

\subsection{General approach}

Let us consider the following portfolio choice problem,

$$
\left\{\begin{array}{l}
\operatorname{Min} \rho(y) \\
\mathbb{E}\left(y z_{\pi}\right) \leqslant e^{r_{f} T} \\
\mathbb{E}(y) \geqslant R \\
y \in Y
\end{array}\right.
$$

where $R>e^{r_{f} T}$ represents the minimum required return. Bearing in mind (2), expression (11) minimizes the risk of a reachable pay off whose global price is not higher than one and whose expected value

\footnotetext{
${ }^{4}$ In fact, the properties above are almost the same as to those used by Rockafellar et al. (2006a) to introduce their Expectation Bounded Risk Measures.

5 The measure of Wang may be defined by

$\rho(y) \quad \int_{0}^{1} \operatorname{VaR}_{t}(y) d g(t)$,

$g:[0,1] \rightarrow[0,1]$ given by

$g(t) \quad \Psi\left(a+\Psi^{1}(t)\right)$

with $a>0$ and $\Psi$ denoting the cumulative function of the standard normal distribution. The author showed that this measure reflects interesting properties in the theory of choice, along the lines of Yaari (1987). A generalization of the Wang transform may be found in Goovaerts and Laeven (2008), where the authors draw on risk measures to price derivative securities.
} 
is at least $R$. Thus it is a standard Risk/Return approach with $\rho$ as the risk measure. Of course, higher quantities of money may be in vested. Since $\rho$ and $\mathbb{E}$ are homogeneous the solution of (11) will be multiplied by $C>0$ if $C$ denotes the value of the quantity to in vest and the first and second constraints become $\mathbb{E}\left(y z_{\pi}\right) \leqslant C e^{r_{f} T}$ and $\mathbb{E}(y) \geqslant R C$, respectively.

The minimization of risk measures is a complex problem that may be addressed by several methods. Among others, the ap proaches of Ruszczynski and Shapiro (2006) or Rockafellar et al. (2006b) appropriately overcome those problems generated by the lack of differentiability of $\rho$. Nevertheless, we will follow the method of Balbás et al. (2009) and, accordingly, we will transform (11) in the new problem

$$
\left\{\begin{array}{l}
\operatorname{Min} \theta \\
\theta+\mathbb{E}(y z) \geqslant 0, \quad \forall z \in \Delta_{\rho} \\
\mathbb{E}\left(y z_{\pi}\right) \leqslant e^{r_{f} T} \\
\mathbb{E}(y) \geqslant R \\
\theta \in \mathbb{R}, y \in Y
\end{array}\right.
$$

$\theta \in \mathbb{R}$ and $y \in L^{2}$ being the decision variables. Following the paper above, (5) allows us to prove that $y$ solves (11) if and only if there exists $\theta \in \mathbb{R}$ such that $(\theta, y)$ solves (12), in which case

$\theta \quad \rho(y)$

holds. Furthermore, with similar arguments to those in Balbás et al. (2009), one can show that Problem

$$
\left\{\begin{array}{l}
\operatorname{Max} \quad e^{r_{f} T} \lambda_{1}+R \lambda_{2} \\
\mathbb{E}\left(y\left(\lambda_{1} z_{\pi} \quad \lambda_{2} \quad z\right)\right) \quad 0, \quad \forall y \in Y \\
z \in \Delta_{\rho}, \quad \lambda_{1} \geqslant 0, \quad \lambda_{2} \geqslant 0
\end{array}\right.
$$

is the dual of (12), $\lambda_{1}, \lambda_{2} \in \mathbb{R}$ and $z \in \Delta_{\rho}$ being the decision variables. The first constraint means that $\lambda_{1} z_{\pi} \quad \lambda_{2} \quad z \in Y^{T}, Y^{T}$ denoting the orthogonal subspace of $Y$. Then it is equivalent to $\varphi_{Y}\left(\begin{array}{lll}\lambda_{1} z_{\pi} & \lambda_{2} & z\end{array}\right) \quad 0$, which, along with $1 \in Y$ and $z_{\pi} \in Y$, lead to the following dual problem

$$
\left\{\begin{array}{l}
\operatorname{Max} \quad e^{r_{f} T} \lambda_{1}+R \lambda_{2} \\
\varphi_{Y}(z) \quad \lambda_{1} z_{\pi} \quad \lambda_{2} \\
z \in \Delta_{\rho}, \lambda_{1} \geqslant 0, \lambda_{2} \geqslant 0
\end{array} .\right.
$$

Proposition 1. $\mathbb{E}\left(\varphi_{Y}(z)\right) \quad \widetilde{E}$ for every $z \in \Delta_{\rho}$.

Proof. Obviously $z \quad \varphi_{Y}(z) \in Y^{T}$, and $1 \in Y$, so $\mathbb{E}\left(\varphi_{Y}(z)\right) \quad \mathbb{E}(z)$. Therefore, the conclusion follows from (6).

Consequently, we can simplify (13). Indeed, taking expecta tions in the first restriction of (13), and taking into account (3) we have

$\lambda_{1} \lambda_{2}+\widetilde{E}$

Thus, changing the variable $\lambda_{2} \quad \lambda, \lambda_{1} \quad \lambda+\widetilde{E}$ we have the following problem equivalent to (13)

$$
\left\{\begin{array}{l}
\operatorname{Max}\left(R \quad e^{r_{f} T}\right) \lambda \quad \widetilde{E} e^{r_{f} T} \\
\varphi_{Y}(z) \quad(\widetilde{E}+\lambda) z_{\pi} \quad \lambda \\
z \in \Delta_{\rho}, \lambda \geqslant 0
\end{array}\right.
$$

Problems (12) and (14) involve the infinite dimensional Hilbert space $L^{2}$. Thus, the absence of the so called "duality gap" is not guar anteed, i.e., the dual optimal value may be strictly lower than the primal one (Luenberger, 1969). To overcome this caveat we have to verify the fulfillment of the Slater qualification, which requires an additional assumption.

Assumption 2. There exists $y \in Y$ such that $\mathbb{E}\left(y z_{\pi}\right) \leqslant e^{r_{f} T}$ and $\mathbb{E}(y)>e^{r_{f} T}$.

Proposition 2. Problem (12) is feasible and satisfies the Slater qual ification, i.e., there exists $(\theta, y) \in \mathbb{R} \times Y$ satisfying the three constraints of (12) as strict inequalities.

Proof. Consider the pay off $y$ satisfying the conditions of Assump tion 2, a positive constant $C<1$, and for $\alpha \in \mathbb{R}, \alpha>0$, take

$\left.\begin{array}{llll}y_{\alpha} & \alpha y & \left(\alpha e^{r_{f} T}\right. & C\end{array}\right)$.

Then, (3) trivially shows that $\mathbb{E}\left(y_{\alpha}\right)$ tends to $\infty$ as so does $\alpha$ whereas $\mathbb{E}\left(y_{\alpha} z_{\pi}\right) \leqslant C$. Hence, we can fix $\alpha$ large enough to guarantee the ful fillment of the second and third constraints as strict inequalities. In addition, the function $\Delta_{\rho} \ni z \rightarrow \mathbb{E}\left(y_{\alpha} z\right) \in \mathbb{R}$ is continuous and $\Delta_{\rho}$ is compact, so taking $\theta>\operatorname{Max}\left\{\mathbb{E}(y z) ; z \in \Delta_{\rho}\right\}$ the first constraint is satisfied as a strict inequality too.

The Slater qualification ensures the absence of duality gap (Luenberger, 1969). Thus, one can give the Strong Duality Theorem below, whose proof is omitted because a similar one may be found in Balbás et al. (2009).

Theorem 3. Suppose that $y^{*} \in L^{p}$ and $\left(\lambda^{*}, z^{*}\right) \in \mathbb{R} \times L^{q}$. Then, they solve (11) and (14) if and only if the following Karush Kuhn Tucker conditions

$$
\left\{\begin{array}{l}
\left(\lambda^{*}+\widetilde{E}\right)\left(\mathbb{E}\left(y^{*} z_{\pi}\right) \quad e^{r_{f} T}\right) \quad 0 \\
\lambda^{*}\left(\mathbb{E}\left(y^{*}\right) \quad R\right) \quad 0 \\
\mathbb{E}\left(y^{*} z_{\pi}\right) \leqslant e^{r_{f} T} \\
\mathbb{E}\left(y^{*}\right) \geqslant R \\
\varphi_{Y}\left(z^{*}\right) \quad\left(\widetilde{E}+\lambda^{*}\right) z_{\pi} \quad \lambda^{*} \\
\mathbb{E}\left(y^{*} \varphi_{Y}\left(z^{*}\right)\right) \leqslant \mathbb{E}\left(y^{*} \varphi_{Y}(z)\right) \quad \forall z \in \Delta_{\rho} \\
\lambda^{*} \geqslant 0, z^{*} \in \Delta_{\rho}
\end{array}\right.
$$

are fulfilled. Moreover, the dual solution is attainable if (11) is bounded, in which case the optimal value of both (11) and (14) becomes $\left(\begin{array}{lll}R & e^{r_{f} T}\end{array}\right) \lambda^{*} \widetilde{E} e^{r_{f} T}$.

\subsection{Cases with unbounded optimal risk or return}

We devote this subsection to illustrating the existence of exam ples leading to meaningless situations from a economic point of view. Surprisingly, some of these examples will involve very important pricing models (for instance, Black and Scholes) and very important risk measures (for instance, $C V a R$ ). Non pathologi cal cases will be analyzed in the next subsection.

We will consider two notions: Compatibility and strong com patibility between the pricing rule $\Pi$ and the risk measure $\rho$.

Definition 1. Balbás and Balbás, 2009The pricing rule $\Pi$ and the risk measure $\rho$ are said to be compatible if there are no sequences

$\left(y_{n}\right)_{n, 1}^{\infty} \subset Y$

such that $e^{r_{f} T} \Pi\left(y_{n}\right) \quad \mathbb{E}\left(y_{n} z_{\pi}\right) \leqslant 0 \quad$ for every $n \in \mathbb{N}$ and $\operatorname{Lim}_{n} \rho\left(y_{n}\right) \quad \infty$.

Next, let us show the existence of pathological situations.

\footnotetext{
${ }^{6}$ Since $\mathbb{E}\left(y_{0} z_{\pi}\right) \quad 1$ and $\mathbb{E}\left(y_{0}\right) \quad 1$ if $y_{0} \quad 1$ is a riskless security, and $\mathbb{E}\left(y e{ }^{r_{f} T} z_{\pi}\right) \leqslant 1$ and $\mathbb{E}\left(y^{r^{r_{f} T}}\right)>1$, Assumption 2 only imposes the existence of a risky security whose expected return is higher than the interest rate.
} 
Theorem 4. $\Pi$ and $\rho$ are not compatible if and only if Problem (11) is unbounded, i.e., if and only if for every $R>e^{r_{f} T}$ the risk level may tend to $\infty$ whereas the expected return is at least $R$.

Proof. Take $y_{0}$ (11) feasible (its existence is guaranteed by Propo sition 2) and the sequence $\left(y_{n}\right)_{n}^{\infty}$ of the definition above. Then, $\mathbb{E}\left(\left(y_{0}+y_{n}\right) z_{\pi}\right) \leqslant \mathbb{E}\left(y_{0} z_{\pi}\right) \leqslant e^{r_{f} T}$ whereas $\rho\left(y_{0}+y_{n}\right) \leqslant \rho\left(y_{0}\right)+\rho\left(y_{n}\right)$ obviously tends to $\infty$. Hence, it is sufficient to show that $\mathbb{E}\left(y_{0}+y_{n}\right) \geqslant R . \mathbb{E}\left(y_{0}+y_{n}\right) \quad \mathbb{E}\left(y_{0}\right)+\mathbb{E}\left(y_{n}\right) \geqslant R+\mathbb{E}\left(y_{n}\right)$. (7) leads to $\mathbb{E}\left(y_{n}\right) \geqslant \frac{1}{E} \rho\left(y_{n}\right) \geqslant 0$ because $\widetilde{E}>0$ and $\rho\left(y_{n}\right) \leqslant 0$ for $n \in \mathbb{N}$ large enough. ${ }^{7}$

Conversely, if $\Pi$ and $\rho$ are compatible then

\section{$\rho(y) \geqslant \widetilde{E} \mathbb{E}\left(y z_{\pi}\right)$}

for every $y \in Y$ (Balbás and Balbás, 2009), and this implies that $\rho(y) \geqslant \widetilde{E} e^{r_{f} T}$ for every $y \in Y$ such that $\mathbb{E}\left(y z_{\pi}\right) \leqslant e^{r_{f} T}$.

Remark 1. There are many examples that fit in the latter theorem. For instance, Balbás and Balbás (2009) have shown that the CVaR, the WCVaR and the DPT are not compatible with the Black and Scholes model and the Heston models, among many other classic pricing models related to derivative securities. All of these cases lead to portfolio choice problems such that there are available strategies whose risk becomes $\infty$ while their expected return becomes as large as desired. Moreover, since

$\operatorname{VaR}_{\mu_{0}}(y) \leqslant \operatorname{CVaR}_{\mu_{0}}(y)$

holds for every level of confidence $\mu_{0} \in(0,1)$ and every $y \in L^{2}$, if we fix $R>e^{r_{f} T}$ then for the Black and Scholes and for the Heston pricing model one can find a sequence of reachable pay offs whose ex pected return remains higher than $R$ while their $V a R_{\mu_{0}}$ tends to $\infty$.

Remark 2. An obvious consequence of Theorems 3 and 4 is that the compatibility of $\Pi$ and $\rho$ is equivalent to the feasibility of (14), i.e., the existence of $\lambda \geqslant 0$ and $z \in \Delta_{\rho}$ such that

$\varphi_{Y}(z) \quad(\widetilde{E}+\lambda) z_{\pi} \quad \lambda$

holds.

The second important notion in this section is the "strong compatibility".

Definition 2. The pricing rule $\Pi$ and the risk measure $\rho$ are said to be strongly compatible if there exist $\lambda>0$ and $z \in \Delta_{\rho}$ such that (16) holds.

The lack of strong compatibility will also lead to pathological situations.

Theorem 5. Suppose that $\Pi$ and $\rho$ are compatible but they are not strongly compatible. Then:

(a) The dual solution $\left(\lambda^{*}, z^{*}\right)$ exists and satisfies $\lambda^{*} \quad 0$.

(b) The (11) optimal value equals $\widetilde{E} e^{r_{f} T}$ and does not depend on $R$.

Proof. Since $\Pi$ and $\rho$ are compatible Theorem 4 shows that (11) is bounded, so Theorem 3 implies the existence of a dual solution $\left(\lambda^{*}, z^{*}\right)$. The lack of strong compatibility implies that $\lambda^{*} \quad 0$, since there are no (14) feasible solutions with strictly positive $\lambda$. More over, (14) makes it obvious that the optimal value equals $\widetilde{E} e^{r_{f} T}$ and does not depend on $R$.

\footnotetext{
${ }^{7}$ Notice that $\widetilde{E} \quad 0$ cannot hold because (7) would imply $\rho(y) \geqslant 0$ for every $y \in L^{p}$,
} and Definition 1 could not hold.
Remark 3. If the lack of strong compatibility occurs, then once again we are facing a meaningless phenomenon from an economic point of view. Indeed, Theorem $5 b$ points out that the minimum risk level will remain constant and equal to $\widetilde{E} e^{r_{f} T}$, while the expected return $R$ may tend to $\infty$. As in the previous case of lack of compatibility, there is no market price of risk either, since the expected return may increase as desired without any increment of risk. The only difference between both scenarios is given by the behavior of the optimal risk level. If there is no compatibility it may go to $\infty$. If there is compatibility but there is no strong compatibility then it remains the same $\left(\widetilde{E} e^{r_{f} T}\right)$.

Next let us see that the lack of strong compatibility frequently holds for complete markets.

Theorem 6. Suppose that for every $\delta>0$

$\mu\left(z_{\pi}<\delta\right)>0$.

If the market is complete $\left(\begin{array}{ll}Y & L^{2}\end{array}\right)$ and $\rho$ is coherent and expectation bounded then $\Pi$ and $\rho$ are not strongly compatible.

Proof. Since the market is complete $\varphi_{Y}$ becomes the identity map. Furthermore, $\widetilde{E} 1$ because $\rho$ is expectation bounded. Therefore, (16) becomes

$z \quad(1+\lambda) z_{\pi} \quad \lambda$

Suppose that $\Pi$ and $\rho$ are strongly compatible and take $\lambda>0$ and $z \in \Delta_{\rho}$ satisfying (18). Given $\delta>0$ one has that

$\mu\left(z_{\pi}<\frac{\delta}{1+\lambda}\right)>0$,

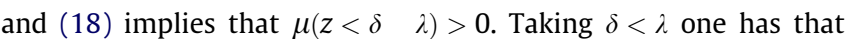
$\mu(z<0)>0$. On the other hand, the coherence of $\rho$ and (8) show that $z \geqslant 0$. Hence, we have a clear contradiction.

Remark 4. There are many examples of complete markets satisfy ing (17). For instance, the Black and Scholes model (Wang, 2000, or Hamada and Sherris, 2003). It may be also proved that the Heston model and other Stochastic Volatility models satisfy (17). All of these models are not strongly compatible with any coherent and expectation bounded risk measure. Very important examples of such a measures are the CCVaR and the Wang measure, among oth ers. For all of these cases there is no market price of risk, and the optimal value of (11) always equals $e^{r_{f} T}$ and does not depend on $R$. In other words, one can construct a portfolio whose expected value is as large as desired and whose risk level remains bounded and constant.

Remark 5. Balbás and Balbás (2009) have shown that the CCVaR and the Black and Scholes model are compatible, but the latter remark points out that they are not strongly compatible. For the Black and Scholes model, and for every level of confidence $\mu_{0} \in(0,1)$, one has that (Balbás and Balbás, 2009)

$\operatorname{CCVaR}_{\mu_{0}}(y) \quad \operatorname{Max}\left\{\operatorname{CVaR}_{\mu_{0}}(y), \Pi(y) e^{r_{f} T}\right\}$.

Since the CCVaR is coherent and expectation bounded, and follow ing Rockafellar et al. (2006a) to construct deviations, one can define the new deviation measure

$\widetilde{N}_{\mu_{0}}(y) \quad \operatorname{Max}\left\{\operatorname{CVaR}_{\mu_{0}}(y), \quad \Pi(y) e^{r_{f} T}\right\}+\mathbb{E}(y)$,

which satisfies the requirements of Assumption 1 for $\widetilde{E} \quad 0$. It is easy to see that $\widetilde{N}_{\mu_{0}}$ and the Black and Scholes model are strongly compatible. Indeed, we could otherwise find a sequence $\left(y_{n}\right)_{n 1}^{\infty} \subset Y$ with $\mathbb{E}\left(y_{n} z_{\pi}\right) \leqslant e^{r_{f} T}$ for every $n \in \mathbb{N},\left(\widetilde{N}_{\mu_{0}}\left(y_{n}\right)\right)_{n 1}^{\infty}$ bounded from above and $\left(\mathbb{E}\left(y_{n}\right)\right)_{n 1}^{\infty}$ going to $\infty$. Therefore, $\left(\operatorname{CCVaR}_{\mu_{0}}\left(y_{n}\right)\right)_{n 1}^{\infty} \quad\left(\widetilde{N}_{\mu_{0}}\left(y_{n}\right)\right)_{n 1}^{\infty} \quad\left(\mathbb{E}\left(y_{n}\right)\right)_{n 1}^{\infty}$ would go to $\infty$. Thus, 
(11) would be unbounded and Theorem 4 would imply that the CCVaR would not be compatible with the Black and Scholes model.

Finally, let us indicate that a similar remark applies if the role of the CVaR is played by the WCVaR.

Remark 6. It is worth noting that the absence of compatibility can not hold if $\rho$ is a deviation measure. Indeed, notice that $\widetilde{E} \quad 0$ in such a case, so (7) points out that $\rho$ does not achieve negative values and, therefore, it cannot tend to $\infty$, i.e., Definition 1 cannot hold.

However, the lack of strong compatibility may still hold. For instance, take the absolute deviation (9) for $p \quad 1$

$\rho(y) \quad \sigma_{1}(y) \quad \mathbb{E}(|\mathbb{E}(y) \quad y|)$.

Then, according to Rockafellar et al. (2006a),

$\Delta_{\rho} \quad\left\{z \quad \mathbb{E}(z) ; z \in L^{\infty},\|z\|_{\infty} \leqslant 1\right\}$.

Therefore, $\Delta_{\rho}$ is obviously composed of (essentially) bounded ran dom variables. In addition, (16) and $\widetilde{E} \quad 0$ lead to

$\begin{array}{lll}z & \lambda z_{\pi} \quad \lambda\end{array}$

for complete markets ( $\varphi_{Y}$ is the identity map). Nevertheless, if $z_{\pi}$ is unbounded (Black and Scholes, Heston etc.), the latter equality im plies that $\lambda \quad 0$, i.e., there is no strong compatibility. Is also interest ing to note that the absolute deviation is the unique $p$ deviation (see (9)) compatible with the Second Order Stochastic Dominance and the standard utility functions (Ogryczak and Ruszczynski, 1999, 2002). Finally, it is also easy to see that the absolute semi deviation ((10), $p \quad 1)$ is not strongly compatible with the Black and Scholes and the Heston models either. It trivially follows from $\sigma_{1}(y) \quad \sigma_{1}(y) / 2 .{ }^{8}$

Remark 7. Finally, it is also remarkable that the standard devia tion is strongly compatible with every pricing rule. Indeed, for $\rho \quad \sigma_{2}$ we have that (Balbás et al., 2009)

$\Delta_{\rho} \quad\left\{z \in L^{2} ; \mathbb{E}(z) \quad 0, \sigma_{2}^{2}(z) \leqslant 1\right\}$.

Then, (3) shows that $\lambda z_{\pi} \quad \lambda \in \Delta_{\rho}$ if $\lambda>0$ is small enough so as to satisfy $\lambda^{2} \sigma_{2}^{2}\left(z_{\pi} 1\right) \leqslant 1$. Furthermore, the equality $E \quad 0$ and $\varphi_{Y}\left(\begin{array}{llll}\lambda z_{\pi} & \lambda\end{array}\right) \quad \lambda z_{\pi} \quad \lambda$ show that Equality (16) holds.

\subsection{Models with a market price of risk}

This subsection will deal with models where the strong compat ibility holds. Thus, henceforth we will assume the following

Assumption 3. There exists strong compatibility between $\Pi$ and $\rho$.

Theorem 7. The dual solution $\left(\lambda^{*}, z^{*}\right)$ exists, does not depend on $R>e^{r_{f} T}$ and satisfies $\lambda^{*}>0$. The (11) and (14) optimal value equals $\left(\begin{array}{lll}R & e^{r_{f} T}\end{array}\right) \lambda^{*} \quad \widetilde{E} e^{r_{f} T}$.

Proof. Assumption 3 and Theorem 4 show that (11) and (14) are bounded and Theorem 3 shows that (14) attains its optimal value. Moreover, it is obvious that this optimal value coincides with the solution of

$\left\{\begin{array}{l}\operatorname{Max} \lambda \\ \varphi_{Y}(z) \quad(\widetilde{E}+\lambda) z_{\pi} \quad \lambda . \\ z \in \Delta_{\rho}, \lambda \geqslant 0\end{array}\right.$

\footnotetext{
${ }^{8}$ It may be proved that the (11)-optimal value is not attainable if we consider the deviations $\sigma_{1}$ or $\sigma_{1}$ and the Black and Scholes model. Hence, the lack of strong compatibility means that one can construct a portfolio whose deviation is as close to zero as desired (but higher than zero) and whose expected return is as large as desired too.
}

The remaining statements are now trivial.

Remark 8. According to (15) the solutions of (11) and (14) are characterized by

$$
\left\{\begin{array}{l}
\mathbb{E}\left(y^{*} z_{\pi}\right) \quad e^{r_{f} T} \\
\mathbb{E}\left(y^{*}\right) \quad R \\
\varphi_{Y}\left(z^{*}\right) \quad\left(\widetilde{E}+\lambda^{*}\right) z_{\pi} \quad \lambda^{*} \\
\mathbb{E}\left(y^{*} \varphi_{Y}\left(z^{*}\right)\right) \leqslant \mathbb{E}\left(y^{*} \varphi_{Y}(z)\right) \quad \forall z \in \Delta_{\rho} \\
\lambda^{*}>0, z^{*} \in \Delta_{\rho}
\end{array}\right.
$$

since $\lambda^{*}+\widetilde{E} \geqslant \lambda^{*}>0$. The two first equalities show that the (11) constraints are saturated, so $R$ is the real expected return of the investment.

Remark 9. If $y^{*}$ solves (11) the absence of duality gap (Theorem 3) and $\lambda^{*}>0$ (Theorem 7) for the dual solution imply that

$\rho\left(y^{*}\right) \quad\left(R \quad e^{r_{f} T}\right) \lambda^{*} \quad \widetilde{E} e^{r_{f} T}$,

and, therefore,

$R \quad \frac{1}{\lambda^{*}}\left(\rho\left(y^{*}\right)+\widetilde{E} e^{r_{f} T}\right)+e^{r_{f} T}$.

One can interpret that $\frac{1}{\lambda^{*}}$ represents the Market Price of Risk, in the sense that there is an affine relationship between optimal risks and returns, and the expected return $R$ increases $\frac{1}{\lambda^{*}}$ units per unit of the risk increment. Due to the analogy with the usual Capital Asset Pric ing Model the affine function (22) will be called Capital Market Line $(C M L)$.

Remark 10. Henceforth fix

$R_{0}>e^{r_{f} T}$

and take $y_{0}^{*}$, solution of (11). Consider $\left(\lambda^{*}, z^{*}\right)$ such that $y_{0}^{*}$ and $\left(\lambda^{*}, z^{*}\right)$ satisfy (21). Then bearing in mind (3) and Proposition 1 it is easy to see that

$\alpha y^{*} \quad\left(\alpha e^{r_{f} T} \quad e^{r_{f} T}\right) \in Y$

and $\left(\lambda^{*}, z^{*}\right)$ also satisfy (21) if $\alpha\left(R_{0} \quad e^{r_{f} T}\right)+e^{r_{f} T}$ replaces $R_{0}$. Since $\alpha\left(R_{0} \quad e^{r_{f} T}\right)+e^{r_{f} T}$ takes all the values within $\left(e^{r_{f} T}, \infty\right)$ as so does $\alpha$ within the interval $(0, \infty)$, it is clear that the dual solution does not depend on $R_{0}$ and the primal one is a combination of $y_{0}^{*}$ and the riskless asset leading to the required expected return.

The remaining efficient portfolios (solutions of (11)) that arise as $R$ varies are combinations of the risk free asset and the benchmark $y_{0}^{*}$. So, for $R>e^{r_{f} T}$ the discussion above shows that the proportion $\alpha$ to invest in the benchmark $y_{0}^{*}$ must satisfy

$R \quad \alpha\left(R_{0} \quad e^{r_{f} T}\right)+e^{r_{f} T}$

which leads to

$\alpha \frac{R \quad e^{r_{f} T}}{R_{0} e^{r_{f} T}}$

and

$1 \propto \frac{R_{0} \quad R}{R_{0} \quad e^{r_{f} T}}$

will be invested in the riskless security.

Every portfolio $y \in Y$ with $\Pi(y) \quad 1$ and $\mathbb{E}(y) \quad R>1$ must be replaced by

$\frac{R \quad e^{r_{f} T}}{R_{0} \quad e^{r_{f} T}} y_{0}^{*}+\frac{R_{0} \quad R}{R_{0} \quad e^{r_{f} T}} e^{r_{f} T} \quad \frac{\mathbb{E}\left(y \quad e^{r_{f} T}\right)}{\mathbb{E}\left(y_{0}^{*} \quad e^{r_{f} T}\right)} y_{0}^{*}+\frac{\mathbb{E}\left(y_{0}^{*} \quad y\right)}{\mathbb{E}\left(y_{0}^{*} \quad e^{r_{f} T}\right)} e^{r_{f} T}$

so as to reach an efficient portfolio with optimal risk level. The opti mal risk level 
$\frac{\mathbb{E}\left(y \quad e^{r_{f} T}\right)}{\mathbb{E}\left(y_{0}^{*} \quad e^{r_{f} T}\right)} \rho\left(y_{0}^{*}\right) \quad \widetilde{E} \frac{\mathbb{E}\left(y_{0}^{*} \quad y\right)}{\mathbb{E}\left(y_{0}^{*} \quad e^{r_{f} T}\right)} e^{r_{f} T}$

will be called systematic risk of $y$, and the remaining risk

$\rho(y) \frac{\mathbb{E}\left(y \quad e^{r_{f} T}\right)}{\mathbb{E}\left(y_{0}^{*} e^{r_{f} T}\right)} \rho\left(y_{0}^{*}\right)+\widetilde{E} \frac{\mathbb{E}\left(y_{0}^{*} \quad y\right)}{\mathbb{E}\left(y_{0}^{*} \quad e^{r_{f} T}\right)} e^{r_{f} T}$

will be called idiosyncratic or specific.

\section{CAMP and APT-like models}

The object of this section is to prove that CAPM and APT like for mulas also hold in the general framework we are dealing with. To this purpose we will consider the portfolio

$y_{1}^{*} \quad \varphi_{\pi}\left(y_{0}^{*}\right) \in \mathcal{L}\left(1, z_{\pi}\right)$,

orthogonal projection of the benchmark $y_{0}^{*}$ on the linear manifold $\mathcal{L}\left(1, z_{\pi}\right)$ generated by the riskless asset and the SDF. Since $y_{1}^{*} \quad y_{0}^{*} \in \mathcal{L}\left(1, z_{\pi}\right)^{T}$, orthogonal subspace of $\mathcal{L}\left(1, z_{\pi}\right)$ (Maurin, 1967), one has that $\mathbb{E}\left(y_{1}^{*} \quad y_{0}^{*}\right) \quad \mathbb{E}\left(\left(\begin{array}{lll}y_{1}^{*} & y_{0}^{*}\end{array}\right) z_{\pi}\right) \quad 0$, which gives

$\mathbb{E}\left(y_{1}^{*}\right) \quad \mathbb{E}\left(y_{0}^{*}\right) \quad R_{0}$

and

$\mathbb{E}\left(y_{1}^{*} z_{\pi}\right) \quad \mathbb{E}\left(y_{0}^{*} z_{\pi}\right) \quad e^{r_{f} T}$.

In particular, $y_{1}^{*}$ is (11) feasible.

Hereafter, the variance of a random variable $y \in L^{2}$ and the covariance between two random variables $y_{1}, y_{2} \in L^{2}$ will be de noted by $\sigma_{2}^{2}(y)$ and $\mathbb{C}\left(y_{1}, y_{2}\right)$, respectively.

In the classic CAPM and APT models one must assume that the market is not risk neutral, which means that the Market Portfolio is not a riskless security (Duffie, 1988). In fact, our Assumption 2 also imposes a non risk neutral market.

Proposition 8. The market is not risk neutral, i.e., the benchmarks $y_{0}^{*}$ and $y_{1}^{*}$ are not riskless securities (are not zero variance). Therefore, the $S D F z_{\pi}$ is not a riskless security either and $\mathcal{L}\left(1, z_{\pi}\right) \quad \mathcal{L}\left(1, y_{1}^{*}\right)$.

Proof. If $y_{0}^{*}$ were riskless security then $y_{0}^{*} \in \mathcal{L}\left(1, z_{\pi}\right)$ and $y_{1}^{*} \quad \varphi_{\pi}\left(y_{0}^{*}\right) \quad y_{0}^{*}$ would be a riskless security too. Thus, let us show that $y_{1}^{*}$ is not a riskless security. Indeed, suppose that $y_{1}^{*}$ is constant. (23) and (27) show that $y_{1}^{*}>e^{r_{f} T}$, and therefore, $\mathbb{E}\left(y_{1}^{*} z_{\pi}\right) \quad y_{1}^{*} \mathbb{E}\left(z_{\pi}\right)$ $y_{1}^{*}$ owing to (3), which contradicts (28).

Furthermore, $y_{1}^{*} \in \mathcal{L}\left(1, z_{\pi}\right)$ points out that $z_{\pi}$ is not a riskless security either since otherwise the dimension of $\mathcal{L}\left(1, z_{\pi}\right)$ would equal one and $y_{1}^{*}$ would have to be risk free. Finally, the equality $\mathcal{L}\left(1, z_{\pi}\right) \quad \mathcal{L}\left(1, y_{1}^{*}\right)$ is already trivial.

Theorem 9 (APT like formula). Suppose that $\left\{y_{1}, y_{2}, \ldots, y_{k}\right\} \subset Y$ is a linearly independent system such that $\mathbb{C}\left(y_{i}, y_{j}\right) \quad 0$ for $i \neq j$. Suppose also that the benchmark $y_{1}^{*}$ satisfies

$y_{1}^{*} \in \mathcal{L}\left(1, y_{1}, y_{2}, \ldots, y_{k}\right){ }^{9}$

Then, for every reachable pay off $y \in Y$ we have that

$y \quad \mathbb{E}\left(y z_{\pi}\right) \quad \sum_{j 1}^{k} \beta_{j}\left(y_{j} \quad \mathbb{E}\left(y_{j} z_{\pi}\right)\right)+\varepsilon_{y}$

and

$\mathbb{E}\left(\begin{array}{ll}y & y z_{\pi}\end{array}\right) \quad \sum_{j 1}^{k} \beta_{j}\left(\mathbb{E}\left(y_{j} \quad y_{j} z_{\pi}\right)\right)$,

\footnotetext{
${ }^{9}$ Notice that this condition is equivalent to $z_{\pi} \in \mathcal{L}\left(1, y_{1}, y_{2}, \ldots, y_{k}\right)$ due to $\mathcal{L}\left(1, z_{\pi}\right) \quad \mathcal{L}\left(1, y_{1}^{*}\right)$.
}

$\varepsilon_{y} \in Y$ satisfying

$\mathbb{E}\left(\varepsilon_{y}\right) \quad 0, \quad \Pi\left(\varepsilon_{y}\right) \quad 0, \quad$ and $\mathbb{C}\left(\varepsilon_{y}, y_{j}\right) \quad 0, \quad j \quad 1,2, \ldots, k$

and $\beta_{j}$ being the regression coefficient

$\beta_{j} \frac{\mathbb{C}\left(y, y_{j}\right)}{\sigma_{2}^{2}\left(y_{j}\right)}$

$j \quad 1,2, \ldots, k$. In particular, if $\Pi(y) \quad \Pi\left(y_{j}\right) \quad 1, j \quad 1,2, \ldots, k$, then

$y \quad e^{r_{f} T} \quad \sum_{j}^{k} \beta_{j}\left(y_{j} \quad e^{r_{f} T}\right)+\varepsilon_{y}$

and

$\mathbb{E}\left(\begin{array}{ll}y & e^{r_{f} T}\end{array}\right) \quad \sum_{j}^{k} \beta_{j}\left(\mathbb{E}\left(y_{j} \quad e^{r_{f} T}\right)\right)$.

Corollary 10 (CAPM like formula). For every reachable pay off $y \in Y$ we have that

$\begin{array}{llll}y & \mathbb{E}\left(y z_{\pi}\right) & \beta\left(y_{1}^{*}\right. & \left.e^{r_{f} T}\right)+\varepsilon_{y}\end{array}$

and

$\mathbb{E}\left(\begin{array}{ll}y & y z_{\pi}\end{array}\right) \quad \beta\left(\mathbb{E}\left(y_{1}^{*} \quad e^{r_{f} T}\right)\right)$,

$\varepsilon_{y} \in Y$ satisfying

$\mathbb{E}\left(\varepsilon_{y}\right) \quad 0, \quad \Pi\left(\varepsilon_{y}\right) \quad 0, \quad$ and $\mathbb{C}\left(\varepsilon_{y}, y_{1}^{*}\right) \quad 0$,

and $\beta$ being the regression coefficient

$\beta \frac{\mathbb{C}\left(y, y_{1}^{*}\right)}{\sigma_{2}^{2}\left(y_{1}^{*}\right)}$.

In particular, if $\Pi(y) \quad 1$, then

$y \quad e^{r_{f} T} \quad \beta\left(y_{1}^{*} \quad e^{r_{f} T}\right)+\varepsilon_{y}$

and

$\mathbb{E}\left(\begin{array}{llll}y & e^{r_{f} T}\end{array}\right) \quad \beta\left(\mathbb{E}\left(y_{1}^{*} \quad e^{r_{f} T}\right)\right)$.

Proof. Let us prove Theorem 9 since Corollary 10 is a trivial conse quence if one bears in mind (2) and (28). Obviously, if $R_{j} \mathbb{E}\left(y_{j}\right), j \quad 1,2, \ldots, k$,

$\left\{1,\left(\frac{y_{j} R_{j}}{\sigma_{2}\left(y_{j}\right)}\right)_{j 1}^{k}\right\} \subset Y$

is an orthonormal system. Thus, the Projection Lemma of Hilbert Spaces (Maurin, 1967) establishes the existence of $\varepsilon_{y} \in \mathcal{L}\left(1, y_{1}, y_{2}, \ldots, y_{k}\right)^{T}$ such that

$y \quad \tilde{\beta}_{0}+\sum_{j 1}^{k} \tilde{\beta}_{j}\left(\frac{y_{j}}{\sigma_{2}\left(y_{j}\right)}\right)+\varepsilon_{y}$,

where

$\tilde{\beta}_{0} \quad \mathbb{E}(y)$

and

$\tilde{\beta}_{j} \mathbb{E}\left(y\left(\frac{y_{j}}{\sigma_{2}\left(y_{j}\right)}\right)\right)$,

$j \quad 1,2, \ldots, k . \varepsilon_{y} \in \mathcal{L}\left(1, y_{1}, y_{2}, \ldots, y_{k}\right)^{T}$ trivially leads to (30), and multiplying by $z_{\pi}$ and taking expectations in (37) one has 
$\tilde{\beta}_{0} \quad \Pi(y) e^{r_{f} T} \quad \sum_{j 1}^{k} \tilde{\beta}_{j}\left(\frac{\Pi\left(y_{j}\right) e^{r_{f} T}}{\sigma_{2}\left(y_{j}\right)} R_{j}\right)$.

Whence (37) becomes

$y \quad \Pi(y) e^{r_{f} T} \quad \sum_{j}^{k} \tilde{\beta}_{j}\left(\frac{y_{j} \quad \Pi\left(y_{j}\right) e^{r_{f} T}}{\sigma_{2}\left(y_{j}\right)}\right)+\varepsilon_{y}$,

which, due to (2), leads to (29) if one takes $\beta_{j} \quad \beta_{j} / \sigma_{2}\left(y_{j}\right), j \quad 1,2, \ldots, k$. Moreover, (31) trivially follows from (38). The remaining expressions, (32) and (33), are now obvious.

Remark 11. Expressions (32) and (33) are clearly similar to those of the classical APT model. They indicate that the real $y \quad e^{r_{f} T}$ and the expected $\mathbb{E}\left(y \quad e^{r_{f} T}\right)$ risk premiums may be given by a family of non correlated factors that generate the benchmark $y_{1}^{*}$ if one adds the riskless asset. One only needs to estimate the systematic risk levels $\beta_{j}$, given by (31), which yield the sensitivity of the secu rity (pay off) $y$ with respect to the $j$ th factor explaining the mar ket. The committed error $\varepsilon_{y}$ has neither correlation with the factors nor with the benchmark $y_{1}^{*}$, and, therefore, it is something specific of the security $y$.

Analogously, (35) and (36) indicate that the real $y \quad e^{r_{f} T}$ and the expected $\mathbb{E}\left(y \quad e^{r_{f} T}\right)$ risk premiums may also be given by the real $y_{1}^{*} \quad e^{r_{f} T}$ and the expected $\mathbb{E}\left(y_{1}^{*} \quad e^{r_{f} T}\right)$ risk premiums of the benchmark $y_{1}^{*}$. The relationship is given by the systematic risk level $\beta$ given by (34). Once again the error $\varepsilon_{y}$ has no correlation with the benchmark $y_{1}^{*}$ and it is specific to the asset/portfolio we are analyzing.

As stated in Remark 10, given $y \in Y$ with $\Pi(y) \quad 1$ one can construct an efficient portfolio with the same expected return but lower risk. (24) and (36) show that the efficient portfolio is

$\beta y_{0}^{*}+(1 \quad \beta) e^{r_{f} T}$,

where $\beta$ is given by (34). The systematic risk (25) of $y$ becomes

$\beta \rho\left(y_{0}^{*}\right) \quad \widetilde{E}(1 \quad \beta) e^{r_{f} T}$,

which is clearly given by $\beta$ once $\rho\left(y_{0}^{*}\right)$ is known, i.e., $\beta$ may be under stood as a measure of the systematic risk.

$\rho$ being a homogeneous, translation invariant and sub additive risk measure implies that

$$
\begin{aligned}
\rho(y) & \rho\left(e^{r_{f} T}+\beta\left(y_{1}^{*} \quad e^{r_{f} T}\right)+\varepsilon_{y}\right) \\
\leqslant & \beta \rho\left(y_{1}^{*}\right) \quad \widetilde{E}(1 \quad \beta) e^{r_{f} T}+\rho\left(\varepsilon_{y}\right) .
\end{aligned}
$$

Since $y_{0}^{*}$ is efficient (28) and (27) prove that

$\rho\left(y_{0}^{*}\right) \leqslant \rho\left(y_{1}^{*}\right)$,

with equality if and only if $y_{1}^{*} y_{0}^{*}$. Thus, bearing in mind (39), if $\beta \geqslant 0$ the specific (26) risk of $y$ will be

$$
\begin{aligned}
& \rho(y) \quad \beta \rho\left(y_{0}^{*}\right)+\widetilde{E}(1 \quad \beta) e^{r_{f} T} \leqslant \beta \rho\left(y_{1}^{*}\right) \quad \widetilde{E}(1 \quad \beta) e^{r_{f} T}+\rho\left(\varepsilon_{y}\right) \\
& \beta \rho\left(y_{0}^{*}\right)+\widetilde{E}(1 \quad \beta) e^{r_{f} T} \\
& \beta\left(\rho\left(y_{1}^{*}\right) \quad \rho\left(y_{0}^{*}\right)\right)+\rho\left(\varepsilon_{y}\right),
\end{aligned}
$$

and, therefore, we have an upper bound for the idiosyncratic risk that depends on $\varepsilon_{y}$ and the difference of risk between both bench marks. The term $\beta\left(\rho\left(y_{1}^{*}\right) \quad \rho\left(y_{0}^{*}\right)\right)$ will vanish if and only if $y_{1}^{*} y_{0}^{*}$.

In the particular case of the Standard Deviation $\rho \quad \sigma_{2}$ (see (9)), if $Y$ is generated by a static approach $(\mathcal{T} \quad\{0, T\}$, only one trading date), it is known that $y_{0}^{*} \in \mathcal{L}\left(1, z_{\pi}\right)$, which obviously im plies that $y_{1}^{*} \quad y_{0}^{*}$, and both the benchmark $y_{0}^{*}$ providing the effi cient portfolios and the one $y_{1}^{*}$ providing the CAPM like formulas (35) and (36) coincide. Then, it may be interesting to characterize those properties leading to an identical situation if $\rho$ is a more general risk measure or deviation and the pricing model may be dynamic.

Remark 12. Consider the dual solution $\left(\lambda^{*}, z^{*}\right)$ that may obtained by solving the linear problem (20). $y_{1}^{*} \quad y_{0}^{*}$ holds if and only if there exist $x_{1}, x_{2} \in \mathbb{R}$ such that $y_{0}^{*} \quad x_{1}+x_{2} z_{\pi}$. Since $\left(y_{0}^{*}, \lambda^{*}, z^{*}\right)$ must satisfy (21) we have that

$$
\begin{cases}x_{1}+x_{2} \mathbb{E}\left(z_{\pi}^{2}\right) & e^{r_{f} T} \\ x_{1}+x_{2} & R_{0}\end{cases}
$$

must hold, which, taking into account Proposition 8 and $0<\sigma_{2}^{2}\left(z_{\pi}\right) \quad \mathrm{E}\left(z_{\pi}^{2}\right) \quad \mathrm{E}\left(z_{\pi}\right)^{2} \quad \mathrm{E}\left(z_{\pi}^{2}\right) \quad 1$, shows that $y_{1}^{*} \quad y_{0}^{*}$ if and only if

$$
y_{0}^{*} \quad \frac{R_{0}\left(1+\sigma_{2}^{2}\left(z_{\pi}\right)\right) \quad e^{r_{f} T}}{\sigma_{2}^{2}\left(z_{\pi}\right)} \quad \frac{R_{0} \quad e^{r_{f} T}}{\sigma_{2}^{2}\left(z_{\pi}\right)} z_{\pi} .
$$

The fulfillment of (40) is easy to verify once $y_{0}^{*}$ has been computed, or by checking whether

$$
\left(\frac{R_{0}\left(1+\sigma_{2}^{2}\left(z_{\pi}\right)\right) \quad e^{r_{f} T}}{\sigma_{2}^{2}\left(z_{\pi}\right)} \quad \frac{R_{0} \quad e^{r_{f} T}}{\sigma_{2}^{2}\left(z_{\pi}\right)} z_{\pi}, \lambda^{*}, z^{*}\right)
$$

satisfies (21).

Despite the fact that the latter remark characterizes the fulfill ment of $y_{1}^{*} y_{0}^{*}$, one can also give other conditions that only re quire solving the linear problem (20).

Theorem 11. Consider the dual solution $\left(\lambda^{*}, z^{*}\right) . y_{1}^{*} \quad y_{0}^{*}$ holds if and only if $\rho\left(z_{\pi}\right) \quad \mathbb{E}\left(z_{\pi} z^{*}\right)$.

Proof. The latter remark states that $y_{1}^{*} \quad y_{0}^{*}$ holds if and only if (40) holds. (21) implies that it is equivalent to the inequality

$$
\begin{aligned}
& \mathbb{E}\left(\left(\frac{R_{0}\left(1+\sigma_{2}^{2}\left(z_{\pi}\right)\right)}{\sigma_{2}^{2}\left(z_{\pi}\right)} \quad e^{r_{f} T} \quad \frac{R_{0} \quad e^{r_{f} T}}{\sigma_{2}^{2}\left(z_{\pi}\right)} z_{\pi}\right) \varphi_{Y}\left(z^{*}\right)\right) \\
& \leqslant \mathbb{E}\left(\left(\frac{R_{0}\left(1+\sigma_{2}^{2}\left(z_{\pi}\right)\right) \quad e^{r_{f} T}}{\sigma_{2}^{2}\left(z_{\pi}\right)} \quad \frac{R_{0}}{\sigma_{2}^{2}\left(z_{\pi}\right)} z_{\pi}^{r_{f} T}\right) \varphi_{Y}(z)\right) \quad \forall z \in \Delta_{\rho}
\end{aligned}
$$

Manipulating, and taking into account Proposition 1, the previous inequality is equivalent to

$\mathbb{E}\left(z_{\pi} \varphi_{Y}\left(z^{*}\right)\right) \geqslant \mathbb{E}\left(z_{\pi} \varphi_{Y}(z)\right) \quad \forall z \in \Delta_{\rho}$.

Since $z_{\pi} \in Y$ and $z \quad \varphi_{Y}(z) \in Y^{T}$ we have $\mathbb{E}\left(z_{\pi} \varphi_{Y}(z)\right) \quad \mathbb{E}\left(z_{\pi} z\right) \forall z \in \Delta_{\rho}$, and the inequality is equivalent to

$\mathbb{E}\left(z_{\pi} z^{*}\right) \geqslant \mathbb{E}\left(z_{\pi} z\right) \forall z \in \Delta_{\rho}$

and the result trivially follows from (5).

Consequently, for the Standard Deviation, which is strongly compatible with every pricing model due to Remark 7, the equality $y_{1}^{*} \quad y_{0}^{*}$ also holds for dynamic approaches.

Corollary 12. If $\rho \quad \sigma_{2}$ then $y_{1}^{*} \quad y_{0}^{*}$.

Proof. Remark 7 shows that there are (14) feasible solutions $(\lambda, z)$ with $\lambda>0$. Hence, if $\left(\lambda^{*}, z^{*}\right)$ solves (14) $\lambda^{*}>0$. Suppose that we prove that

$\left(\lambda^{*}, z^{*}\right) \quad\left(\frac{1}{\sigma_{2}\left(z_{\pi}\right)}, \frac{1}{\sigma_{2}\left(z_{\pi}\right)} z_{\pi} \frac{1}{\sigma_{2}\left(z_{\pi}\right)}\right)$

solves (14). Then 


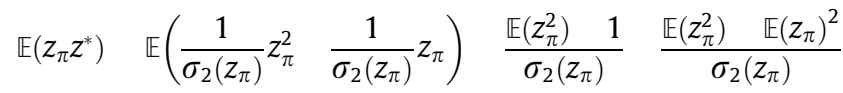

$$
\begin{aligned}
& \frac{\sigma_{2}^{2}\left(z_{\pi}\right)^{2}}{\sigma_{2}\left(z_{\pi}\right)} \quad \sigma_{2}\left(z_{\pi}\right) \quad \sigma_{2}\left(z_{\pi}\right)
\end{aligned}
$$

and the latter theorem applies. Let us now see (41). Since $z^{*} \quad \varphi_{Y}\left(z^{*}\right)$ and $\varphi_{Y}\left(z^{*}\right) \quad \lambda^{*} z_{\pi} \quad \lambda^{*}$ are orthogonal the Pythagorean Theorem of Hilbert Spaces (Maurin, 1967) and (6) lead to

$$
\begin{aligned}
\sigma_{2}^{2}\left(z^{*}\right) \quad\left\|z^{*}\right\|_{2}^{2} & \left\|z^{*} \quad \varphi_{Y}\left(z^{*}\right)\right\|_{2}^{2}+\left\|\varphi_{Y}\left(z^{*}\right)\right\|_{2}^{2} \geqslant\left\|\lambda^{*} z_{\pi} \quad \lambda^{*}\right\|_{2}^{2} \\
& \sigma_{2}^{2}\left(\lambda^{*} z_{\pi} \quad \lambda^{*}\right) .
\end{aligned}
$$

Moreover, since $1 \geqslant \sigma_{2}^{2}\left(z^{*}\right)$ due to (19), $\left\|z^{*} \quad \varphi_{Y}\left(z^{*}\right)\right\|_{2}^{2}>0$ would lead to $1>\left(\lambda^{*}\right)^{2} \sigma_{2}^{2}\left(z_{\pi} 1\right)$. Then for $\alpha>1$ and small enough $\alpha \lambda^{*}\left(z_{\pi} \quad 1\right)$ would have zero expectation and a variance lower than one, i.e., $\alpha \lambda^{*}\left(z_{\pi} 1\right.$ 1) would be (14) feasible due to (19). Since $\alpha \lambda^{*}>\lambda^{*}$ because $\alpha>1$ and $\lambda^{*}>0$, we have a contradiction because $\left(\lambda^{*}, z^{*}\right)$ cannot solve (14).

$$
\text { Consequently, } z^{*} \quad \varphi_{Y}\left(z^{*}\right) \quad 0 \text {, and } z^{*} \quad \varphi_{Y}\left(z^{*}\right) \quad \lambda^{*} z_{\pi} \quad \lambda^{*} \text {. As }
$$
above, $1>\left(\lambda^{*}\right)^{2} \sigma_{2}^{2}\left(z_{\pi} \quad 1\right)$ cannot hold, so

$$
\left(\lambda^{*}\right)^{2} \sigma_{2}^{2}\left(z_{\pi} \quad 1\right) \quad 1,
$$

which ends the proof.

\section{Conclusions}

This paper deals with the general portfolio choice problem and the classic APT and CAPM models when risk levels are given by risk measures beyond the variance. We include in the analysis expecta tion bounded risk measures, coherent risk measures and general deviation measures. This seems a topic of interest since the vari ance presents some drawbacks. For instance, it is not always com patible with the Second Order Stochastic Dominance. With respect to the reachable pay offs, we focus on the pricing rule and the SDF rather than the distribution of the returns of the available securi ties. Consequently, this analysis may apply for both static and dy namic pricing models.

First of all, we give general optimality conditions, despite the le vel of generality for both risks and prices. Secondly, we introduce new notions such as strong compatibility between prices and risks. Surprisingly, the lack of (strong) compatibility leads to unbounded portfolio choice problems, despite the fact that obtaining economic interpretations for that is complex. Nevertheless, the lack of (strong) compatibility holds for very important risk measures (VaR, CVaR, WCVaR, CCVaR, DPT, Wang, absolute deviation, absolute downside semi deviation, etc.) and pricing models (Black Scholes, Heston, other derivative linked pricing models, etc.). Thirdly, we also characterize and analyze models with a market price of risk, which may also involve dynamic pricing models. A CML is gener ated and two major benchmarks are introduced. Finally, we pres ent APT and CAPM like developments, and they do not modify the classic definitions of the betas. On the contrary, systematic risks only depend on correlations with the factors/market, whereas idiosyncratic risks and noises have null correlation with the fac tors/market.

\section{Acknowledgments}

Research partially carried out during the visit to Concordia Uni versity, Montreal, Canada. The authors thank the Mathematics and Statistics Department for their great hospitality.

The authors also thank "RD_Sistemas SA", “CAM (Spain) Grant s 0505/tic/000230", and "MEyC (Spain) Grant SEJ2006 15401 C04", for their partial support. The usual caveat applies.

\section{References}

Alexander, S., Coleman, T.F., Li, Y., 2006. Minimizing CVaR and VaR for a portfolio of derivatives. Journal of Banking \& Finance 30, 538-605.

Annaert, J., Van Osselaer, S., Verstraete, B., 2009. Performance evaluation of portfolio insurance strategies using stochastic dominance criteria. Journal of Banking \& Finance 33, 272-280.

Artzner, P., Delbaen, F., Eber, J.M., Heath, D., 1999. Coherent measures of risk. Mathematical Finance 9, 203-228.

Balbás, A., Balbás, R., 2009. Compatibility between pricing rules and risk measures: The CCVaR. Revista de la Real Academia de Ciencias, RACSAM 103, 251-264.

Balbás, A., Balbás, R., Garrido, J., 2010. Extending pricing rules with general risk functions. European Journal of Operational Research 201, 23-33.

Balbás, A., Balbás, B., Heras, A., 2009. Optimal reinsurance with general risk measures. Insurance: Mathematics and Economics 44, 374-384.

Barbarin, J., Devolder, P., 2005. Risk measure and fair valuation of an investment guarantee in life insurance. Insurance: Mathematics and Economics 37, 297323.

Brown, D., Sim, M., 2009. Satisfying measures for analysis of risky positions. Management Science 55, 71-84.

Calafiore, G.C., 2007. Ambiguous risk measures and optimal robust portfolios. SIAM Journal of Optimization 18, 853-877.

Chamberlain, G., Rothschild, M., 1983. Arbitrage, factor structure and meanvariance analysis of large assets. Econometrica 51, 1281-1304.

Cheridito, P., Tianhui, L., 2009. Risk measures on Orlicz hearts. Mathematical Finance 19, 189-214.

Duffie, D., 1988. Security Markets: Stochastic Models. Academic Press.

Föllmer, H., Schied, A., 2002. Convex measures of risk and trading constraints. Finance \& Stochastics 6, 429-447.

Goovaerts, M., Kaas, R., Dhaene, J., Tang, Q., 2004. A new classes of consistent risk measures. Insurance: Mathematics and Economics 34, 505-516.

Goovaerts, M., Laeven, R., 2008. Actuarial risk measures for financial derivative pricing. Insurance: Mathematics and Economics 42, 540-547.

Hamada, M., Sherris, M., 2003. Contingent claim pricing using probability distortion operators: method from insurance risk pricing and their relationship to financial theory. Applied Mathematical Finance 10, 19-47.

Horvàth, J., 1966. Topological Vector Spaces and Distributions, vol. I. Addison Wesley, Reading, MA.

Johnston, M., 2009. Extending the Basel II approach to estimate capital requirements for equity investments. Journal of Banking \& Finance 33, 11771185.

Luenberger, D.G., 1969. Optimization by Vector Spaces Methods. John Wiley \& Sons.

Maurin, K., 1967. Methods of Hilbert spaces. PWN-Polish Scientific Publishers.

Miller, N., Ruszczynski, A., 2008. Risk-adjusted probability measures in portfolio optimization with coherent measures of risk. European Journal of Operational Research 191, 193-206.

Nakano, Y., 2004. Efficient hedging with coherent risk measure. Journal of Mathematical Analysis and Applications 293, 345-354.

Ogryczak, W., Ruszczynski, A., 1999. From stochastic dominance to mean risk models: semideviations and risk measures. European Journal of Operational Research 116, 33-50.

Ogryczak, W., Ruszczynski, A., 2002. Dual stochastic dominance and related mean risk models. SIAM Journal of Optimization 13, 60-78.

Quaranta, A.G., Zaffaroni, A., 2008. Robust optimization of conditional value at risk and portfolio selection. Journal of Banking \& Finance 32, 2046-2056.

Rockafellar, R.T., Uryasev, S., Zabarankin, M., 2006a. Generalized deviations in risk analysis. Finance \& Stochastics 10, 51-74

Rockafellar, R.T., Uryasev, S., Zabarankin, M., 2006b. Optimality conditions in portfolio analysis with general deviations measures. Mathematical Programming, Series B 108, 515-540.

Rockafellar, R.T., Uryasev, S., Zabarankin, M., 2006c. Master funds in portfolio analysis with general deviation measures. Journal of Banking \& Finance 30, 743-778.

Rockafellar, R.T., Uryasev, S., Zabarankin, M., 2007. Equilibrium with investors using a diversity of deviation measures. Journal of Banking \& Finance 31, 3251-3268.

Ruszczynski, A., Shapiro, A., 2006. Optimization of convex risk functions. Mathematics of Operations Research 31, 433-452.

Schied, A., 2007. Optimal investments for risk- and ambiguity-averse preferences: A duality approach. Finance \& Stochastics $11,107-129$.

Staum, J., 2004. Fundamental theorems of asset pricing for good deal bounds. Mathematical Finance 14, 141-161.

Stoyanov, S.V., Rachev, S.T., Fabozzi, F.J., 2007. Optimal financial portfolios. Applied Mathematical Finance 14, 401-436.

Wang, S.S., 2000. A class of distortion operators for pricing financial and insurance risks. Journal of Risk and Insurance 67, 15-36.

Yaari, M.E., 1987. The dual theory of choice under risk. Econometrica 55, 95115.

Zakamouline, V., Koekebbaker, S., 2009. Portfolio performance evaluation with generalized Sharpe ratios: beyond the mean and variance. Journal of Banking \& Finance 33, 1242-1254.

Zhiping, C., Wang, Y., 2008. Two-sided coherent risk measures and their application in realistic portfolio optimization. Journal of Banking \& Finance 32, 2667-2673. 